\title{
A global factor in the Hermann grid illusion or an artifact?
}

\author{
MARC CHADERJIAN, JOHN M. PRICE, and THEODORE E. PARKS \\ University of California, Davis, California
}

\begin{abstract}
In the first of the present experiments, subjects were required to estimate the strength of the Hermann grid illusion in grids containing various numbers of intersections even though those grids were not actually presented. The positive relationship found by Wolfe (1984) for real grids was, nevertheless, replicated. It is argued that this suggests that a response bias might have been the source of his effect (although other possibilities are also noted). In addition, in a second experiment, subjects who were not aware of the fact that grid size was being manipulated (i.e., between subjects) showed no consistent effect of that factor, thus supporting the same suggestion.
\end{abstract}

The Hermann grid illusion- that is to say, the occurrence, for example, of illusory dark spots within the intersections of white "streets" separated by a set of larger black squares-is widely held to be, at least in large part, the product of "local" mechanisms: of activities related to the areas within and immediately surrounding each individual intersection (Hering, 1964; see also, e.g., Baumgartner, 1960, and Uttal, 1973). Thus, although certain Hermann grid phenomena and explanations seem to require or allow for nonlocal mechanisms or effects (Laming, 1992; Troscianko, 1983), it would seem prudent to treat such exceptions as unusual claims requiring unusually strong evidence for their acceptance.

One such claim that seems to fail such a test is the claim that increasing the number of blocks (and, therefore, the number of intersections) in a Hermann grid pattern results in an increase in the strength of the lightness illusion seen therein (Wolfe, 1984). For example, to anticipate, in the first of the present experiments we repeated his experiment in as much detail as his description of it allowed, but actually presented only one grid (as an "anchor" for the rating scale) and found evidence that strongly suggests that the effect found by Wolfe might have been an artifact of his procedure.

\section{EXPERIMENT 1}

\section{Method}

Subjects. Forty-eight upper division psychology students participated in this study. All subjects were kept naive as to Wolfe's (1984) experiment (including his results and conclusion) and to our true purpose.

Correspondence should be addressed to T. E. Parks, Department of Psychology, University of California, Davis, CA 95616-8686 (e-mail: teparks@ucdavis.edu).
Materials and Procedure. Subjects were given a high-contrast photocopy of a $7 \times 7$ (36 intersection) grid, each block being $8.5 \mathrm{~mm}$ square and separated by streets $3 \mathrm{~mm}$ wide. Attached to this photocopy was a second sheet containing the instructions for the rating task and a response form.

The rating methodology and instructions reproduced as closely as possible those used by Wolfe (1984), the difference being that no grids (except for the $7 \times 7$ standard) were ever actually presented to the subjects. Instead, the subjects were asked to rate the "strength" of the illusory gray spots for grids of various sizes that they were to imagine. Specif ically, like Wolfe's subjects, they were told to use a rating scale on which a value of "7" was assigned to the level of illusory gray that they saw in the $7 \times 7$ grid. They were also told (again, as in Wolfe's study) that a rating of " 1 " would indicate no illusion, ratings of " 2 " to " 6 " would indicate an illusion that was weaker ("lighter, or less gray") than the one in the $7 \times 7$ grid, and that a rating of " 8 " or " 9 " would be allowed if the illusion in a particular imagined grid was stronger ("darker, or more gray") that the one in the $7 \times 7$ grid (see, for comparison, Wolfe, 1984, p. 35).

Following these general points the subjects were asked to imagine an " $y \times y$ " grid, and, according to the rating scale, assign a number to that imagined grid's illusory strength. Specifically, they were to do so on the basis of only one of the imagined grid's intersections; the point being to attempt to avoid ratings based on the expected number of illusory spots rather than their expected individual strength.

The " $y$ " values in this study were 3,5 , or 9 (thus they were to imagine grids containing 4, 16, and 64 intersections). The order of these three values for a particular subject corresponded to a randomly chosen row of a $3 \times 3$ Latin square, all orders being used equally often across subjects.

\section{Results and Discussion}

As can be seen in Figure 1, despite the absence of a set of physical grids and, therefore, of any physical variation in the number of intersections, a strong effect of that variable was obtained, mean ratings for the smallest through the largest grids being 3.7, 5.6, and 8.1, respectively. Variance around these points was quite small and the effect is well beyond generally accepted levels of chance $[F(2,94)=99.44, p<.001]$. 


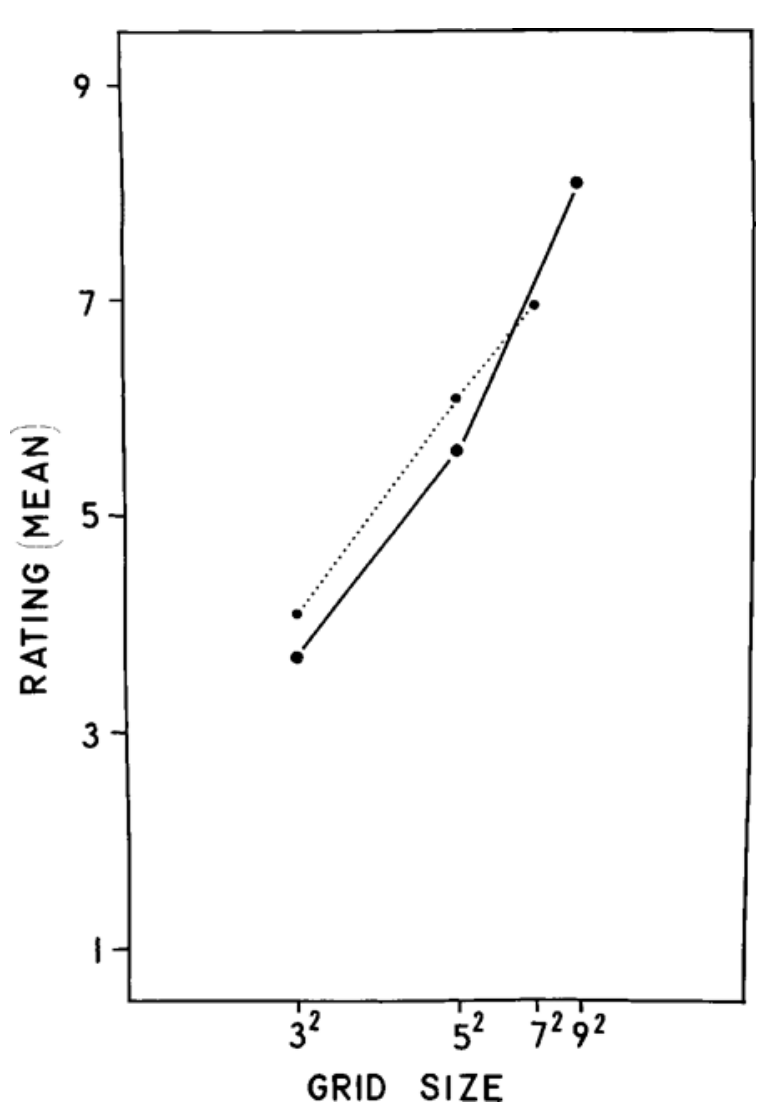

Figure 1. Mean ratings of illusion strength. The small points were derived from Wolfe (1984), where grid size was physically varied, and the large points represent data from subjects who actually viewed only a $7 \times 7$ grid. It should be noted that, unlike Wolfe, the present authors did not ask subjects to reiterate the rating assigned to the $7 \times 7$ grid. Instead, a grid that had more intersections than those used by Wolfe was included in an attempt to extend the range of his effect.

More than the mere production of a reliable effect in the same direction as Wolfe's, Figure 1 also shows (quite dramatically) that these subjects managed to duplicate closely the precise means obtained by him (Wolfe, 1984, Figure $3 a)$. Such precision requires explanation, and there are conflicting possibilities.

One possibility that must be considered is that both Wolfe's (1984) results and the present replication reflect "genuine" effects (that is to say, effects that actually do occur within precepts and within images, respectively). More specifically, it may be that an effect occurred within the imagined grid which "mimicked" an effect seen within physical grids, much as, for example, the effect of physical eccentricity on visual acuity is mimicked in the results of imaginary acuity tests (Finke, 1986). The difficulty for such a proposal in the present case, however, is that the evidence that the effects produced by imagined grids mimic those of real grids is only convincing if the effect of grid size, like the effect of eccentricity, is well estab- lished for real stimulation in the first place. If this is not the case, finding the same effect without physical exposure to the relevant variable must, instead, cast doubt on the "real" effect, itself. That is, in the absence of strong supporting evidence, it is obvious that any pattern of reports that occurs whether or not one performs the physical manipulation that supposedly causes it, cannot be attributed with any certainty to the efficacy of that physical manipulation within perception, itself, when it is performed; subjects may simply report what they believe they should. By contrast, no one doubts the evidence for the effect of physical eccentricity; for one thing, in that case the causes of the effect are well known and understood.

It follows that Wolfe's results cannot be taken as a strong argument for this particular global effect on the Hermann grid illusion.

\section{EXPERIMENT 2}

If Wolfe's (1984) results were, in fact, based on a response bias, it would follow that his effect should disappear if subjects were not aware that grid size was the independent variable. Thus, finding his effect under such conditions would argue against our explanation. We tested this possibility in a second experiment (but instead of using a rating method-which would make it difficult to keep subjects in ignorance-we used a matching technique instead).

\section{Method}

Subjects. Sixty naive introductory psychology students served as subjects, receiving extra credit toward their course grade as compensation.

Stimuli and Apparatus. Four Hermann grid stimuli (one to be assigned to each group of 15 subjects) were produced on high contrast paper, using a copy camera; each grid consisted of three, five, seven, or nine rows and columns of black "blocks," each of which was $1.9 \mathrm{~cm}$ on a side and was separated from those adjacent by "streets" of $0.7 \mathrm{~cm}$. Under the room illumination used in the experiment, the black areas had a reflected luminance of $8 \mathrm{~cd} / \mathrm{m}^{2}$ and the white areas reflected $167 \mathrm{~cd} / \mathrm{m}^{2}$, as measured by a Pritchard Model 1980 photometer. A hole to the right side of each grid, at a distance of $2.8 \mathrm{~cm}$ and in line with its center, allowed viewing of an adjustable gray scale. This hole was $1.0 \mathrm{~cm}$ in diameter, to approximate the size of the illusory gray spots at the intersections of the streets of the grid.

The gray scale was produced photographicall y using Kodak Kodabromide paper of grade F-2, which was slowly uncovered lengthwise in the presence of light, developed normally, and dried without ferrotyping. Measurements made under the room illumination used in the experiment indicated that the strips, indeed, presented a smooth increase in reflectance from a reflected luminance of 140 to one of $32 \mathrm{~cd} / \mathrm{m}^{2}$.

The subject was seated at a distance of approximately $1.5 \mathrm{~m}$ from the display so that the width of each street subtended $.5^{\circ}$ of visual angle. In this position, the distance between the intersections nearest to the opening to a gray strip was $4^{\circ}$ of visual angle.

Procedure. The subjects were instructed to match the gray that appeared in the aforementioned hole to the illusory gray seen at the upper and lower of the two intersections of the first vertical street 
inside the grid at the corners of the nearest block. At the start of each trial, the gray strip was placed randomly at either the darkest or the lightest point and the subject was allowed as much time as was desired to move from there to a satisfactory match. This procedure was then repeated four times for the same grid.

\section{Results and Discussion}

The mean settings obtained for the smallest through largest grids were $102,90,107$, and $95 \mathrm{~cd} / \mathrm{m}^{2}$, respectively $[F(3,46)=1.29]$. Thus, rather than the consistent effect found with Wolfe's (1984) rating procedure, no consistent increasing effect of grid size was evident (at least, for subjects, like these, who were unaware of the independent variable). For example, the lightest setting was given for the second-to-largest grid.

Taken along with the results of the first experiment, this strongly suggests, again, that Wolfe's (1984) results may well have been artifactual. This is not to say that this hypothesis has been disproved, but only that his evidence is questionable at this time. ${ }^{1}$

\section{REFERENCES}

BAUmgarTnER, G. L. (1960). Indirekte Grosenbestimmung der receptiven Felder der Retina beim Menschen mittels der Hermannschen
Gittertauschung. Pflïgers Archiv für die gesamte Physiologie des Menschen und der Tiere, 272, 21-22.

FInKE, R. A. (1986). Mental imagery and the visual system. Scientific American, 254(3), 88-95.

Hering, E. (1964). Outlines of a theory of the light sense. In L. M. Hurvich \& D. Jameson (Trans.). Cambridge, MA: Harvard University Press. Original work published 1878.

LAMING, D. (1992). Springer's lines and Hermann's grid. Opthalmic \& Physiological Optics, 12, 178-182.

Troscianko, T. (1983). Local and global processes in the Hermann grid illusion [Abstract]. Perception, 12, A23.

UtTal, W. R. (1973). The psychobiology of sensory coding. Potomac, MD: Erlbaum.

Wolfe, J. M. (1984). Global factors in the Hermann grid illusion. Perception, 13, 33-40.

\section{NOTE}

1. It is important to note that any reader who is interested in possibly observing Wolfe's effect should be aware of two potential contaminating factors (in addition to expectations): the increased number of illusory spots (as opposed to possible change in their darkness) and the possibility that the greater number of spots increased the probability that one or more of them would be particularly strong. The essential point is that neither of these requires that the darkness of any particular illusory spot be influenced by remote events.

(Manuscript accepted for publication October 31, 1997.) 